\title{
Mass fractal characteristics of silica sonogels as determined by small-angle $x$-ray scattering and nitrogen adsorption
}

\author{
D. A. Donatti, D. R. Vollet, A. Ibañez Ruiz, A. Mesquita, and T. F. P. Silva \\ Unesp-Universidade Estadual Paulista, IGCE, Departamento de Física, P.O. Box 178 CEP, 13500-970 Rio Claro, São Paulo, Brazil
}

(Received 16 April 2004; revised manuscript received 22 July 2004; published 13 January 2005)

\begin{abstract}
A sample series of silica sonogels was prepared using different water-tetraethoxysilane molar ratio $\left(r_{w}\right)$ in the gelation step of the process in order to obtain aerogels with different bulk densities after the supercritical drying. The samples were analyzed by means of small-angle x-ray-scattering (SAXS) and nitrogen-adsorption techniques. Wet sonogels exhibit mass fractal structure with fractal dimension $D$ increasing from $\sim 2.1$ to $\sim 2.4$ and mass-fractal correlation length $\xi$ diminishing from $\sim 13 \mathrm{~nm}$ to $\sim 2 \mathrm{~nm}$, as $r_{w}$ is changed in the nominal range from 66 to 6 . The process of obtaining aerogels from sonogels and heat treatment at $500{ }^{\circ} \mathrm{C}$, in general, increases the mass-fractal dimension $D$, diminishes the characteristic length $\xi$ of the fractal structure, and shortens the fractal range at the micropore side for the formation of a secondary structured particle, apparently evolved from the original wet structure at a high resolution level. The overall mass-fractal dimension $D$ of aerogels was evaluated as $\sim 2.4$ and $\sim 2.5$, as determined from SAXS and from pore-size distribution by nitrogen adsorption, respectively. The fine structure of the "secondary particle" developed in the obtaining of aerogels could be described as a surface-mass fractal, with the correlated surface and mass-fractal dimensions decreasing from $\sim 2.4$ to $\sim 2.0$ and from $\sim 2.7$ to $\sim 2.5$, respectively, as the aerogel bulk density increases from $0.25\left(r_{w}=66\right)$ up to $0.91 \mathrm{~g} / \mathrm{cm}^{3}\left(r_{w}=6\right)$.
\end{abstract}

DOI: 10.1103/PhysRevB.71.014203 PACS number(s): 61.10.Eq, 61.43.Hv, 81.20.Fw, 82.70.Gg

\section{INTRODUCTION}

A large variety of glass and glass ceramics has been obtained by the sol-gel process from the hydrolysis of tetraethoxysilane (TEOS). ${ }^{1}$ Sonohydrolysis is an alternative method to promote the solventless hydrolysis of the alkoxide. Wet sonogels frequently exhibit structures with massfractal characteristics, consisting of a continuous solid network imbibed in a liquid phase. The development of massfractal structures in wet sonogels has been well established by means of a kinetic study at different temperatures of the aggregation process of very small silica particles produced in the TEOS sonohydrolysis. ${ }^{2}$ Supercritical drying (aerogels) and evaporation drying (xerogels) are usual methods in the production of dried gels. Most of the original structure of the wet sonogels is expected to be preserved in the solvent exchange and supercritical drying in the obtaining process of aerogels.

A comparative study using small-angle x-ray scattering (SAXS) and nitrogen adsorption has been carried out in the characterization of sonogel-derived aerogels. ${ }^{3}$ An independent method to probe the mass-fractal structure of aerogels, exclusively from the pore-size distribution as obtained from the nitrogen adsorption isotherms, has been suggested so the results were found in good agreement with SAXS results. ${ }^{3}$

In this work, a sample series of silica sonogels was prepared using different water-tetraethoxysilane molar ratios $\left(r_{w}\right)$ in the gelation step of the process in order to obtain aerogels with different bulk densities after the supercritical drying. Interesting structural properties associated with the wet sonogels and the evolution of such a structure in the obtaining of the sonogel-derived aerogels have been inferred from the mass-fractal characteristics as determined from the small-angle $\mathrm{X}$-ray scattering (SAXS) and nitrogen adsorption data.

\section{EXPERIMENT}

Samples of sonogels were prepared from the sonohydrolysis of mixtures of $25 \mathrm{ml}$ of tetraethoxysilane (TEOS), $8 \mathrm{ml}$ of distilled and dionized water, and $5 \mathrm{ml}$ of $0.1 \mathrm{~N} \mathrm{HCl}$ as a catalyst. The $p \mathrm{H}$ of the hydrolysis mixture was about 1.5-2.0. The sonohydrolysis was carried out during $10 \mathrm{~min}$ under a constant power $(60 \mathrm{~W})$ of ultrasonic radiation. The resulting hydrolyzed sol was diluted in different quantities of water in order to obtain final aerogels with different densities, and the sonication was continued for 2 min for complete homogenization. The added water quantity was changed to get samples with the final water-TEOS molar ratio $\left(r_{w}\right)$ according to the nominal values $r_{w}=6,10,19,25,40$, and 66 . The $p \mathrm{H}$ of the samples was adjusted to 4.5 by addition of $\mathrm{NH}_{4}(\mathrm{OH})$, and the samples were cast in sealed containers and kept under saturated conditions for 20 days at $40{ }^{\circ} \mathrm{C}$ for gelation and aging. The wet sonogels were studied by smallangle x-ray scattering (SAXS). Aerogels were obtained by a two-step process: first, the liquid phase of the wet sonogels was exchanged by pure ethanol at room temperature; second, ethanol was exchanged by liquid $\mathrm{CO}_{2}$ in an autoclave followed by supercritical $\mathrm{CO}_{2}$ extraction. Aerogels prepared with $r_{w}=66,40$, and 25 were monoliths, and those prepared with minor $r_{w}$ values were monolithic slices; the cracking was found increasing as $r_{w}$ decreasing. Sample aerogels were heat treated at a temperature of $500{ }^{\circ} \mathrm{C}$ under atmospheric conditions for about $10 \mathrm{~h}$ and studied by SAXS and nitrogen-adsorption techniques.

The SAXS experiments were carried out using synchrotron radiation with a wavelength $\lambda=0.1608 \mathrm{~nm}$. The beam was monochromatized by a silicon monochromator and collimated by a set of slits defining a pin-hole geometry. A one-dimensional position-sensitive $\mathrm{x}$-ray detector was used 


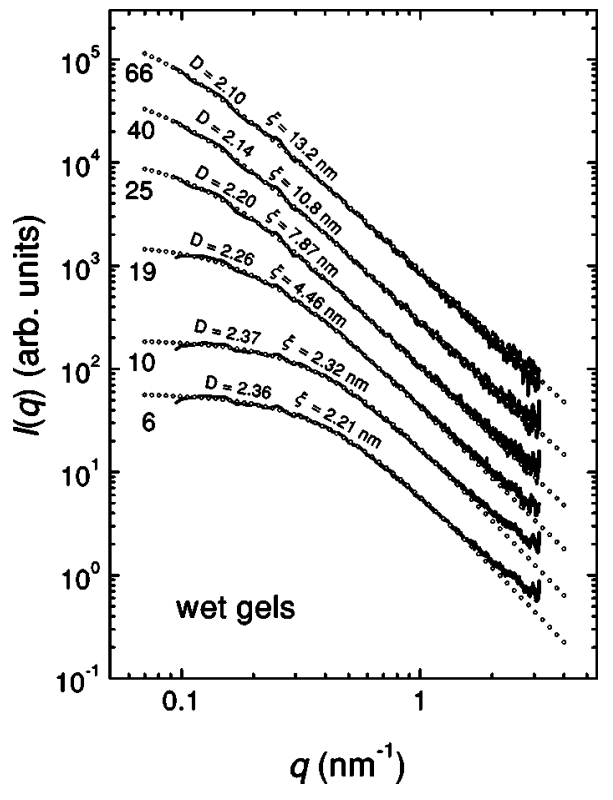

FIG. 1. SAXS intensity as a function of the modulus of scattering vector $q$ for wet sonogels with $r_{w}$ ranging according to the nominal values $66,40,25,19,10$, and 6 , as indicated. The small circle lines are fittings of the mass-fractal approach [Eq. (1)] to the experiment data.

to record the SAXS intensity $I(q)$ as a function of the modulus of the scattering vector $q=(4 \pi / \lambda) \sin (\theta / 2)$, where $\theta$ is the scattering angle. The experimental set allowed one to get SAXS data from $q_{0}=0.095 \mathrm{~nm}^{-1}$ to $q_{m}=3.2 \mathrm{~nm}^{-1}$ with a resolution of $\Delta q=4.98 \times 10^{-3} \mathrm{~nm}^{-1}$. The data were corrected by the parasitic scattering and sample attenuation and normalized by the intensity of the incident beam. The parasiticscattering correction has been carried out by measuring the scattering from an empty cell for both aerogels and wet sonogels. The maximum experimental value $q_{m}=3.2 \mathrm{~nm}^{-1}$ in the measured $q$ range, which corresponds to a Bragg distance of $1.8 \mathrm{~nm}$, is not high enough to resolve the liquid-phase structure in wet sonogels, so no correction by the solvent scattering has been made on the wet sonogels.

Nitrogen adsorption isotherms were obtained at liquid nitrogen temperature using a commercial pore and surface analyzer apparatus (ASAP 2010 Micromeritics). The data were analyzed for BET-specific surface $\left(S_{\mathrm{BET}}\right)$, total pore volume per mass unit $\left(V_{p}\right)$, as the total adsorbed volume of nitrogen at a single point close to the saturation pressure, and porevolume distribution, as determined by using the classical Kelvin equation and the Harkins and Jura model for cylindrical pores. ${ }^{4}$

\section{RESULTS}

Figure 1 shows the SAXS intensity $I(q)$ as a function of the modulus of scattering vector $q$ of the sonogels as measured under wet conditions. Figure 2 shows the same type of curves for the corresponding aerogels after supercritical extraction of $\mathrm{CO}_{2}$ and heat treatment at $500{ }^{\circ} \mathrm{C}$ for $10 \mathrm{hr}$.

The mass fractal characteristics of the sonogels can be recognized by a typical power-law decrease on $q$ of the

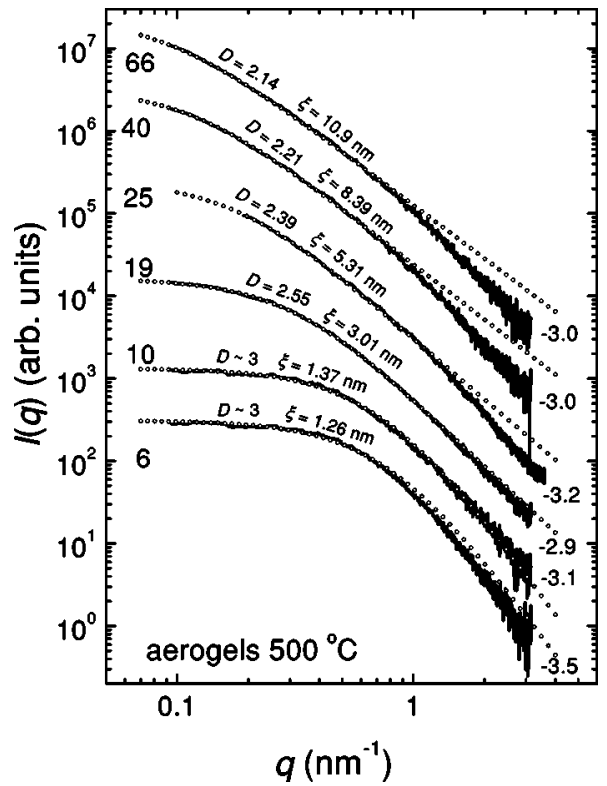

FIG. 2. SAXS intensity as a function of the modulus of scattering vector $q$ for aerogels obtained after the supercritical drying of the sonogels of Fig. 1 and heat treatment at $500{ }^{\circ} \mathrm{C}$. The small circle lines are fittings of the mass-fractal approach [Eq. (1)] to the experiment data. The slopes of the experimental curves at high $q$ associated to Porod's region are indicated in each case.

SAXS intensity in a $q$ range given by $\xi \gg q^{-1} \gg a$, where $\xi$ is the characteristic length of the fractal structure and $a$ is the characteristic length of the primary particles composing the structure. ${ }^{5}$ The SAXS intensity departs from the power-law regime at low- $q$ values due to the finite-correlation range $\xi$ of the mass-fractal structure, thus, an approach can be cast as ${ }^{6}$

$$
I(q)=A \sin [(D-1) \arctan (q \xi)] /\left(1+q^{2} \xi^{2}\right)^{(D-1) / 2}(D-1) q \xi,
$$

where $D$ is the mass-fractal dimension of the structure, a physically acceptable value between $1<D<3$, and $A$ is a constant for a given $D$ and $\xi$.

Figure 1 shows that the curves of the SAXS intensity of the wet sonogels are well fitted by Eq. (1) in practically all the studied $q$ range. The lack of crossover to Porod's law ${ }^{7}$ $\left(I(q) \sim q^{-4}\right)$ at high- $q$ region reveals that the system is a mass fractal up to almost the molecular level because the minor length scale probed by the present SAXS study, through $1 / q_{m} \sim 0.3 \mathrm{~nm}$, corresponds to a Bragg distance of $\sim 1.8 \mathrm{~nm}$. The structural parameters of the fitting process $D$ and $\xi$ through Eq. (1) are shown in Table I together with the fractality range expressed as the ratio $\xi / a, a$ being the characteristic length of the primary silica particle, assumed as $a \sim 0.3 \mathrm{~nm} \sim 1 / q_{m}$. A positive deviation from Eq. (1) is apparent at high- $q$ in the SAXS curves of the samples with $r_{w}=6$ and 10 in Fig. 1. Probably, wet aerogels with low- $r_{w}$ values present some structure at a high resolution level accounting for an additional contribution to SAXS at high $q$.

In the SAXS curves of the aerogels (Fig. 2), after the $\mathrm{CO}_{2}$ supercritical extraction and heat treatment at $500{ }^{\circ} \mathrm{C}$, it was found that Eq. (1) fits generally well the SAXS intensity at 
TABLE I. Structural parameters as determined by SAXS for wet gels and aerogels.

\begin{tabular}{rccccccc}
\hline \hline & \multicolumn{3}{c}{ Wet gels } \\
\cline { 2 - 7 }$r_{w}$ & $D$ & $\xi(\mathrm{nm})$ & $\xi / a$ & $D$ & $\xi(\mathrm{nm})$ & $\xi / a$ & $\alpha$ \\
\hline 66 & $2.10 \pm 0.02$ & $13.2 \pm 0.1$ & $\sim 40$ & $2.14 \pm 0.01$ & $10.9 \pm 0.1$ & $\sim 10$ & $3.0 \pm 0.1$ \\
40 & $2.14 \pm 0.02$ & $10.8 \pm 0.1$ & $\sim 32$ & $2.21 \pm 0.02$ & $8.39 \pm 0.07$ & $\sim 8$ & $3.0 \pm 0.1$ \\
25 & $2.20 \pm 0.03$ & $7.87 \pm 0.07$ & $\sim 24$ & $2.39 \pm 0.02$ & $5.31 \pm 0.05$ & $\sim 7$ & $3.2 \pm 0.1$ \\
19 & $2.26 \pm 0.03$ & $4.46 \pm 0.05$ & $\sim 13$ & $2.55 \pm 0.03$ & $3.01 \pm 0.03$ & $\sim 7$ & $2.9 \pm 0.1$ \\
10 & $2.37 \pm 0.04$ & $2.32 \pm 0.03$ & $\sim 5$ & $\sim 3$ & $1.4 \pm 0.1$ & $?$ & $3.1 \pm 0.2$ \\
6 & $2.36 \pm 0.04$ & $2.21 \pm 0.03$ & $\sim 4$ & $\sim 3$ & $1.3 \pm 0.1$ & $?$ & $3.5 \pm 0.3^{\mathrm{a}}$ \\
\hline \hline
\end{tabular}

${ }^{a}$ More properly, it was found increasing from $\sim 3.1$ to $\sim 3.8$ in the fitted range.

the low- and medium- $q$ regions. At the high- $q$ region, it was generally found that the SAXS intensity presents a negative deviation from the Eq. (1), crossing over to a distinct powerlaw decrease on $q$ as $I(q) \sim q^{-\alpha}$. The fitting of Eq. (1) was found to be insensitive to the power law decaying at the high- $q$ region in the case of aerogels with $r_{w}=66,40$, and 25, for the SAXS intensity from those samples was found to be too much greater at low $q$ when compared to that at high $q$ to be equally weighted in the least-squares method. The exponent $\alpha$ was found (Table I), in general, quite close to the value 3 in all cases, except for $r_{w}=6$ when $\alpha$ was found to be $\sim 3.5$. The typical value $\alpha \sim 3$ is rather small to be associated with the Porod regime $\left(I(q) \sim q^{-4}\right)$, so the system presents some structure at a high resolution level. This fine structure could be associated with an extremely rough boundary space-filling fractal surface. ${ }^{5}$ The analysis of the fractal characteristics when $\alpha \sim 3$ may be a complex matter. ${ }^{8}$ As we will see, this fine structure at a high resolution level is also apparent from the nitrogen adsorption study.

Table I shows the SAXS structural parameters of the mass fractal $D$ and $\xi$ obtained for the aerogels with $r_{w}=66,40,25$, and 19 , together with the ratio $\xi / a$, for this set of samples in which the crossover to the power-law decaying $I(q) \sim q^{-\alpha}$ at high $q$ is apparent. The fitting process of Eq. (1) to the aerogels with $r_{w}=10$ and 6 have yielded physically meaningless values for $D(D>3)$. Then, we restricted $D$ to the maximum acceptable value 3 and proceeded in determining $\xi$. However, the significance and correctness of the parameters resulting from the use of Eq. (1) to fit the data of systems with fractal dimension close to 3 (mass or surface) are discussable. ${ }^{8}$

Figure 3 shows the nitrogen-adsorption isotherms as obtained at liquid-nitrogen temperature for the studied aerogels. Table II shows the bulk density $\rho$ and the BET-specific surface area $S_{\mathrm{BET}}$ of the aerogels. $\rho$ was evaluated from the adsorption data using the total pore volume per mass unit $V_{p}$ through the relation $(1 / \rho)=\left(1 / \rho_{a}\right)+V_{p}$, assuming the value $2.2 \mathrm{~g} / \mathrm{cm}^{3}$ for the skeletal density $\rho_{a}$, as frequently quoted for fused silica. It has been pointed out ${ }^{9}$ that, in the case of low-density aerogels, the adsorption method applies stress on the sample during measurement leading to an underestimation of pore volume. This seems not to be the case for our samples of aerogels produced from sonogels due to the good agreement, in general, found between the bulk densities measured from direct mass and volume determinations and from the adsorption data.
Figure 4 shows the curves of the incremental pore volume as a function of the pore width $2 r$ for the studied aerogels. The pore-size distribution curves (PSD) were obtained from the adsorption isotherms (Fig. 3) through a routine using the classical Kelvin equation and the Harkins and Jura model for cylindrical pores. ${ }^{4}$ The pore width $2 r$ in Fig. 4 corresponds to the diameter of the cylindrical pore in the Harkins and Jura model. The resolution of the pore width probed by the Kelvin equation is about $1.4 \mathrm{~nm}$, and the maximum pore width that can be probed by nitrogen adsorption is about $200 \mathrm{~nm}$. In general, the shape of the PSD curves is very similar for all aerogels, except that the curves are magnified and shifted toward the macropore region, as $r_{w}$ is increased. The pore width at the maximum in the PSD curves is also shifted toward the macropore region. The aerogel with $r_{w}=66$ presents maximum in the PSD curve at a pore width not too far from the upper limit of the applicability of the nitrogen-adsorption technique.

Although the pore mean size and the pore volume increase accentually with $r_{w}$ (Fig. 4), the BET-specific surface

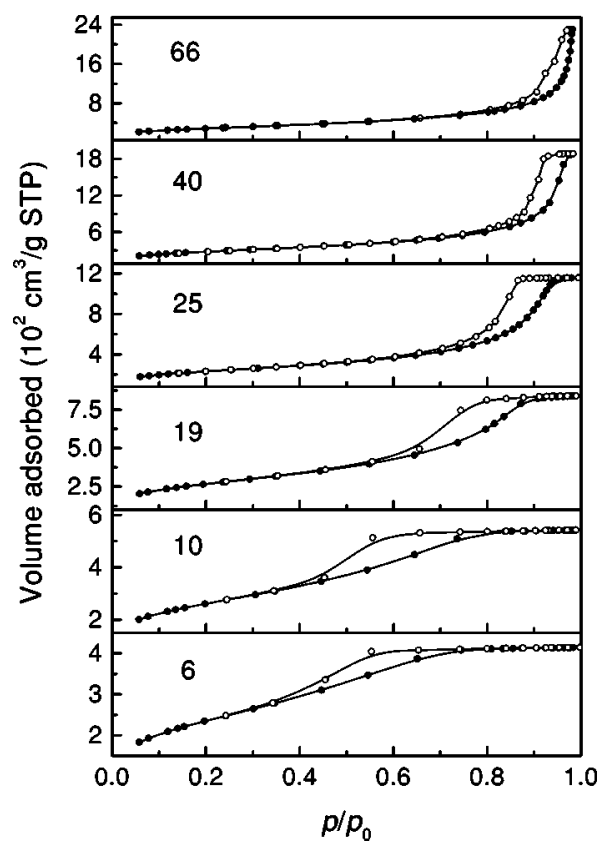

FIG. 3. Nitrogen-adsorption isotherms carried out at liquidnitrogen temperature for the aerogels of Fig. 2. The open-circle points correspond to the desorption branch. 
TABLE II. Structural parameters as determined from nitrogen adsorption for sonogel-derived aerogels treated at $500{ }^{\circ} \mathrm{C}$.

\begin{tabular}{lcccccc}
\hline \hline$r_{w}$ & $\rho\left(\mathrm{cm}^{3} / \mathrm{g}\right)$ & $S_{\mathrm{BET}}\left(10^{2} \mathrm{~m}^{2} / \mathrm{g}\right)$ & $l_{s}(\mathrm{~nm})$ & $D$ & $2 r_{\xi}(\mathrm{nm})$ & $2 r_{\xi} / 2 r_{a}$ \\
\hline 66 & $0.25 \pm 0.01$ & $10.3 \pm 0.1$ & $1.7 \pm 0.1$ & $2.24 \pm 0.03$ & $110 \pm 8$ & $\sim 5$ \\
40 & $0.30 \pm 0.01$ & $10.1 \pm 0.1$ & $1.8 \pm 0.1$ & $2.22 \pm 0.03$ & $61 \pm 6$ & $\sim 5$ \\
25 & $0.44 \pm 0.01$ & $8.32 \pm 0.08$ & $2.2 \pm 0.1$ & $2.33 \pm 0.03$ & $35 \pm 4$ & $\sim 5$ \\
19 & $0.57 \pm 0.02$ & $9.66 \pm 0.09$ & $1.9 \pm 0.1$ & $2.41 \pm 0.03$ & $19 \pm 2$ & $\sim 4$ \\
10 & $0.77 \pm 0.02$ & $9.42 \pm 0.08$ & $1.9 \pm 0.1$ & $2.50 \pm 0.04$ & $9.2 \pm 0.8$ & $\mathrm{a}$ \\
6 & $0.91 \pm 0.03$ & $9.46 \pm 0.08$ & $1.9 \pm 0.1$ & $2.55 \pm 0.04$ & $8.2 \pm 0.6$ & $\mathrm{a}$ \\
\hline \hline
\end{tabular}

aust one mass-fractal regime associated to the micropore region.

area $S_{\mathrm{BET}}$ shows practically a constant value, just increasing very slightly with $r_{w}$ (Table II). This means that the solid network of the aerogels is formed by silica particles, which are very similarly independent on the porosity. Table II shows the average size values of the silica particles $\left(l_{s}\right)$ that form the solid network of aerogels as evaluated by $l_{s}=4 V_{s} / S_{\mathrm{BET}}$, as an analogy with the definition of the BET pore mean size, where $V_{s}=[(1-\phi) / \phi] V_{p}$ is the solid volume and $\phi$ is the pore volume fraction. Most of the values for $l_{s}$ were found in the range between about $1.9 \mathrm{~nm}$ and $1.7 \mathrm{~nm}$, the values lightly diminishing with $r_{w}$.

\section{DISCUSSION}

As a general result from the SAXS, $\xi$ and $\xi / a$ decrease while $D$ increases with the diminution of $r_{w}$, as for wet sonogels as aerogels. The process of obtaining aerogels from sonogels and heat treatment at $500{ }^{\circ} \mathrm{C}$ increases the massfractal dimension $D$, diminishes the characteristic length $\xi$ of the fractal structure, and shortens the fractal range $\xi / a$, apparently for the evolution of the structure at a high resolution level, as if a new secondary structured particle were to be created. The characteristics of such a fine structure could be better understood from the results of the nitrogen adsorption.

To compare the SAXS data with nitrogen adsorption data, we employed an earlier proposed $\operatorname{method}^{3}$ for the generation of a mass-fractal structure from the PSD curve by a sequence of approximants, starting from a homogeneous solid with

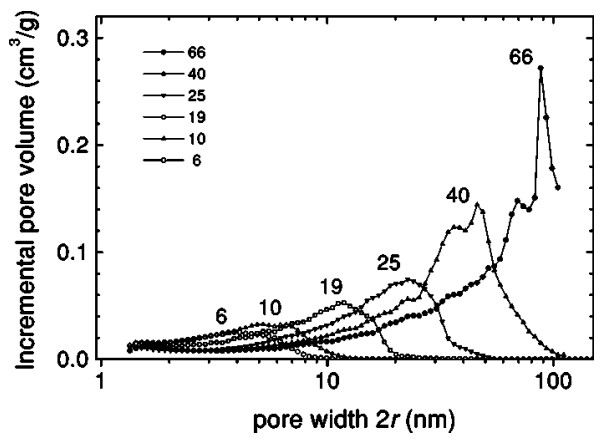

FIG. 4. Pore-volume distribution as obtained by the Kelvin equation and the classical Harkins and Jura model for cylindrical pores from the nitrogen-adsorption isotherms of the aerogels of Fig. 3 . density equal to that of the fused silica $\rho_{a} \sim 2.2 \mathrm{~g} / \mathrm{cm}^{3}$ and probing the resulting bulk density $\rho(r)$, as each incremental pore volume per mass unit $\delta V_{p}\left(r_{i}\right)$ is incorporated to the structure. The process can be cast as

$$
\frac{1}{\rho(r)}=\frac{1}{\rho_{a}}+\sum_{r_{i}=0}^{r_{i}=r} \delta V_{p}\left(r_{i}\right) .
$$

For a mass-fractal structure the bulk density $\rho_{\xi}$ can be cast ${ }^{6}$ as

$$
\rho_{\xi}=\rho_{a}(\xi / a)^{D-3},
$$

where $\xi$ is the characteristic length of the fractal structure and $a$ the characteristic length of the homogeneous silica particle. Then, the evaluation of $\rho(r)$ in the range $a \leqslant r \leqslant \xi$ for such a system should yield

$$
\rho(r)=\rho_{a}(r / a)^{D-3} .
$$

Figure 5 shows $\rho(r)$ for the studied aerogels, in a log-log scale with the structure scale length (as probed by the pore width $2 r$ ), as evaluated through Eq. (2) from the pore volume distribution shown in Fig. 4. Accordingly, the density $\rho(r)$ tends to a constant value equal to the bulk density $\rho$ (Table II) as the pore width $2 r$ increases up to the maximum value $2 r_{\xi}$ in each $r_{w}$-case. The value $2 r_{\xi}$ was found in good agreement with the value $2 \pi \xi$, the Bragg distance associated to the correlation length $\xi$ as probed by SAXS (see Fig. 5). The agreement between the values $2 r_{\xi}$ and $2 \pi \xi$ is not so good for the sample with $r_{w}=66$, for the maximum length $2 \pi / q_{0}$ experimentally probed by SAXS (at the minimum $q_{0}$ ) is minor than the value $2 r_{\xi}$ determined from nitrogen adsorption. Then, the bulk density $\rho$ of Table II could be assigned to $\rho_{\xi}$ [Eq. (3)] and the pore width $2 r_{\xi}$ properly associated to the Bragg distance $2 \pi \xi$ in each case. As a consequence, the overall mass-fractal dimension $D$ of the structure can be probed through Eq. (3) using the values of $\rho_{\xi}$ (or $\rho$ in Table II) and the maximum pore width $2 r_{\xi}$ (adsorption) or the characteristic length $\xi$ (SAXS). Figure 6 shows the plot of $\rho_{\xi}$ versus the pore width $2 r_{\xi}$ (and the Bragg distance $2 \pi \xi$ ) in a $\log -\log$ scale together with the linear fitting of Eq. (3) to the data. From the slope $D-3$ of the straight line fitted in each case, we have found $D=2.51 \pm 0.03$ from adsorption and $D=2.41 \pm 0.03$ from SAXS as a measure of the overall massfractal dimension $D$. The overall value $\sim 2.4$ obtained by SAXS is among the values obtained by fitting Eq. (1) to the 


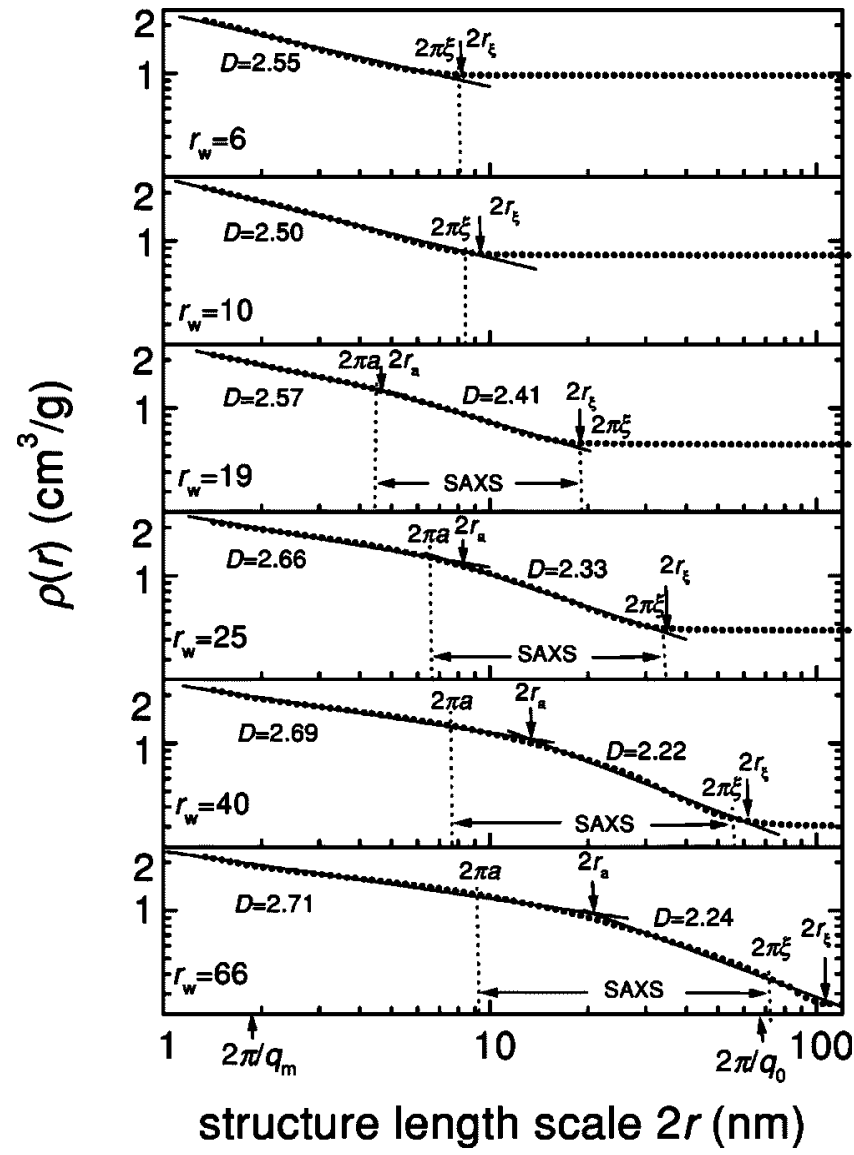

FIG. 5. The bulk density $\rho(r)$ as a function of the structure scale length (as probed by the pore width $2 r$ ) evaluated through Eq. (2) with the PSD curves in Fig. 4. The correlations between the structure scale length as probed by adsorption $\left(2 r_{\xi}\right.$ and $\left.2 r_{\mathrm{a}}\right)$ and by the Bragg distance from SAXS $(2 \pi \xi$ and $2 \pi a)$, associated with the fitting range of the mass-fractal approach [Eqs. (4) and/or (1)], are indicated in each case.

SAXS intensity curves of the aerogels (Table I) (except for the $r_{w}=6$ and 10, as mentioned). Vacher et al. ${ }^{6}$ have found $D=2.40 \pm 0.03$ from small-angle neutron scattering in a series of non-sonogel-derived silica aerogels with different densities.

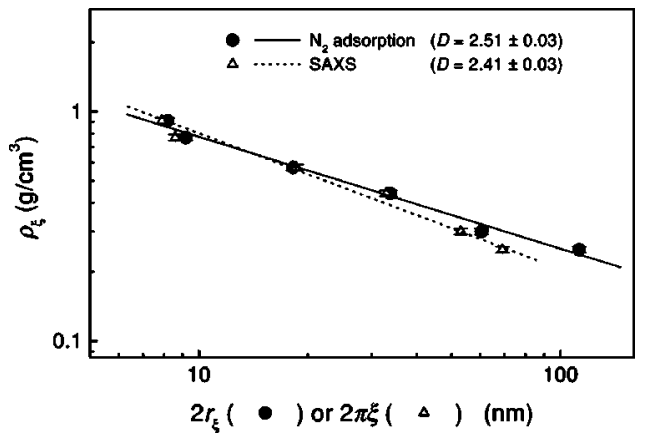

FIG. 6. Probing the mass-fractal dimension $D$ of the overall structure through $\rho_{\xi}=\rho_{\mathrm{a}}(\xi / a)^{D-3}$ using $\rho$ (or $\rho_{\xi}$ ) given in Table II together with the maximum pore width $2 r_{\xi}$ (from adsorption) and the Bragg distance associated with the correlation distance $\xi$ (from SAXS).
According to Eq. (4), the plot in a log-log scale of $\rho(r)$ with the pore width $2 r$ should be a straight line with slope $D-3$. Figure 5 shows that Eq. (4) fits reasonably well to the experimental $\rho(r)$ in a first interval of $2 r$ associated to the mesopore region, between the maximum value $2 r_{\xi}$ and an intermediary value $\sim 2 r_{a}$, for the samples with $r_{w}=66,40$, 25 , and 19 . The values of $D$ obtained from the slopes $D-3$ of the straight lines plotted in the range $2 r_{\xi}-2 r_{a}$ (Fig. 5 and Table II), including the superior limit $2 r_{\xi}$, are in good agreement with those corresponding, as determined by SAXS through Eq. (1) (Table I), to the same set of samples $\left(r_{w}=66,40,25\right.$, and 19).

Figure 5 also shows a second mass-fractal regime associated with the micropore region, in the range between $\sim 2 r_{a}$ and practically the inferior limit of the nitrogen-adsorption pore resolution $(\sim 1.4 \mathrm{~nm})$, for the samples with $r_{w}=66,40$, 25 , and 19. The slopes $D-3$ of the straight lines fitting Eq. (4) for this set of samples yield values for $D$ all in the range 2.7-2.6, the values decreasing from the sample with $r_{w}=66$ to that with $r_{w}=19$ (Fig. 5). The crossover at $\sim 2 r_{a}$, on passing from the mass fractal associated to the mesopore region to that associated to the micropore region, is shifted in direction to the micropore region, as $r_{w}$ diminishes from 66 to 19. The values of $D$ of both fractal regimes gradually approximate one to the other as $r_{w}$ varies from 66 to 19. Particularly for $r_{w}=19$, the crossover at $\sim 2 r_{a}$ is almost nonapparent (Fig. 5). For the sample with $r_{w}=10$ and $r_{w}=6$ the crossover readily disappears, giving place to a single mass-fractal regime with $D \sim 2.5$. Evidently, the mass-fractal structure associated with the micropore region as inferred from adsorption is associated with the fine structure developed in the aerogels as observed from SAXS.

Figure 5 shows the correlations between the SAXS and adsorption data in the description of both observed fractal regimes. The crossover between them has been assigned to $2 r_{a}$ for adsorption and $2 \pi a$ for SAXS. As pointed out, the values of $D$ and of the upper limit $2 r_{\xi}$ of the mass fractal associated to the mesopore region are in good agreement as measured by both techniques (Figs. 2 and 5, Table I and II), for samples with $r_{w}=66,40,25$, and 19. Quantitatively, the values of $D$ associated with the micropore region, which have been found between 2.5 and 2.7 (including all samples) by adsorption (Fig. 5), are not in agreement with the values of $\alpha$ associated with the SAXS high- $q$ power-law decaying $I(q) \sim q^{-\alpha}$, which have been found all approximately equal to 3 for samples with $r_{w}=66,40,25,19$, and 10, and equal to 3.5 for $r_{w}=6$ (Fig. 2). Such an apparent disagreement could be explained if we consider all three types of fractals, as in a surface-mass-pore fractal. ${ }^{10}$

The intensity from a surface-mass-pore fractal obey ${ }^{10}$ the power law $I(q) \sim q^{-\alpha}$, with

$$
\alpha=2\left(D_{m}+D_{p}\right)-6-D_{s},
$$

where $D_{s}, D_{m}$, and $D_{p}$ is the fractal dimension of the surface, mass, and pore space, respectively. For a surface fractal, $D_{m}=D_{p}=3$, it reduces to the Bale-Schmidt ${ }^{11}$ scattering $I(q) \sim q^{6-D_{s}}$; for a mass fractal, $D_{s}=D_{m}$ and $D_{p}=3$, it reduces 
TABLE III. Fractal dimensions of the surface-mass fractal of the fine structure associated with the micropore region of aerogels treated at $500{ }^{\circ} \mathrm{C}$.

\begin{tabular}{lccc}
\hline \hline$r_{w}$ & $D_{m}$ & $\alpha$ & $D_{s}$ \\
\hline 66 & $2.71 \pm 0.05$ & $3.0 \pm 0.1$ & $2.4 \pm 0.2$ \\
40 & $2.69 \pm 0.05$ & $3.0 \pm 0.1$ & $2.4 \pm 0.2$ \\
25 & $2.66 \pm 0.05$ & $3.2 \pm 0.1$ & $2.1 \pm 0.2$ \\
19 & $2.57 \pm 0.05$ & $2.9 \pm 0.1$ & $2.2 \pm 0.2$ \\
10 & $2.50 \pm 0.05$ & $3.1 \pm 0.2$ & $1.9 \pm 0.3$ \\
6 & $2.55 \pm 0.05$ & $3.1-3.8$ & $\sim 2^{\mathrm{a}}$ \\
\hline \hline
\end{tabular}

${ }^{\mathrm{a}} D_{m}$ and $D_{s}$ seem to be only partially correlated through Eq. (5).

to $I(q) \sim q^{-D_{m}}$; and for a pore fractal, $D_{s}=D_{p}$ and $D_{m}=3$, it reduces to $I(q) \sim q^{-D_{p}}$.

We assume that $D_{p}=3$, since the pore-volume fraction $\phi$ is greater than the mass-volume fraction in aerogels. For instance, the sample with $r_{w}=6$ has the highest density among the studied aerogels and presents $\phi \sim 0.60$. So, the fine micropore structure should be a surface-mass fractal to present mass-fractal dimension between 2.5-2.7 (Fig. 5) and account for the exponent $\alpha$ in Table I because $\alpha=2 D_{m}-D_{s}$ for $D_{p}=3$ in Eq. (5). Table III shows the values of the correlated fractal dimensions $D_{m}$, as read from the plots in Fig. 5, and $D_{s}$, as evaluated from Eq. (5) with $D_{p}=3$ for the samples with $r_{w}=66,40,25,19$, and 10 .

According to Table III, the correlated surface fractal dimension $D_{s}$ of the surface-mass fractal associated to the fine structure of the aerogels decreases from $\sim 2.4$ to $\sim 2.0$ while the correlated mass-fractal dimension decreases from 2.7 to 2.5, as $r_{w}$ changes from 66 to 10 . The evaluation of $D_{s}$ for the sample with $r_{w}=6$, using $D_{m}=2.55$ (Fig. 5) and $\alpha=3.5$ (Table I), yields $D_{s} \sim 1.6$, a physically unaccepted value for the surface fractal dimension. However, as mentioned, the curve associated to the final high- $q$ decaying of the SAXS intensity of the sample with $r_{w}=6$ in the plot of Fig. 2, is more properly a nonlinear curve, instead a fairly straight line as in the case of the other samples. The exponent $\alpha$ was estimated changing from about 3.1 to 3.8 in the range of the linear fitting. Then, $D_{m}$ and $D_{s}$ seem have left to be strongly correlated through Eq. (5), so the intensity at the high- $q$ decaying seems to be a result of two contributions: a surfacemass fractal [correlated through Eq. (5)] contribution, predominant at not so high $q$, and a perfectly smooth surface contribution, predominant at very high $q$.

Finally, very fine structural modifications in the secondary structured particle occurring with variations in $r_{w}$ as measured by the surface-mass fractal model could account for that just small variations found in the average size of the solid silica particles $l_{s}$ as shown in Table II. In addition, the increase of the secondary particle size with the increase of $r_{w}$, as well probed by $2 r_{a}$ (or $2 \pi a$ ) in Fig. 5 for $r_{w}=19,25$,
40 , and 66 , together with the small variations found in the average size of the solid silica particles $l_{s}$ in Table II suggest that nitrogen adsorption is, in fact, resolving most of fine structure of the secondary particles.

\section{CONCLUSIONS}

Wet sonogels exhibit a mass-fractal structure with fractal dimension $D$ ranging from $\sim 2.1$ to $\sim 2.4$ and a mass-fractal correlation length $\xi$ ranging from $\sim 13$ to $\sim 2 \mathrm{~nm}^{2}$, as the water/TEOS molar ratio $\left(r_{w}\right)$ used in gelation step of the process is changed in the nominal interval from 66 to 6 .

The process of obtaining aerogels from sonogels and heat treatment at $500{ }^{\circ} \mathrm{C}$ increases the mass-fractal dimension $D$, diminishes the characteristic length $\xi$ of the fractal structure, and shortens the fractal range at the micropore side for the formation of a secondary structured particle, apparently evolved from the original wet mass-fractal structure at a high resolution level.

The fundamental role of the increase of $r_{w}$ on the structure of resulting aerogels is to increase the pore mean size and the pore volume, without, however, substantially modifying the average size of the silica particles forming the solid network of the aerogels. The domains and the characteristics of the mass fractal built up by the primary silica particles were found influenced by the porosity.

In the case of intermediate density aerogels, the values of $D$ and of the mass-fractal domain associated with the mesopore region were correspondingly found in reasonable agreement when determined by SAXS or by nitrogen adsorption. The determination of the inferior limit of the mass-fractal domain from both techniques was found in less agreement in the case of low-density aerogels. For high-density aerogels, the employed SAXS mass-fractal approach does not apply suitably because of the absence of most of the mesopore structure, while the nitrogen adsorption method accounts practically for the mass fractal associated with the micropore structure of the secondary structured particles.

The overall mass-fractal dimension $D$ as evaluated from the bulk density of aerogels as a function of the structure characteristic size was found to be $\sim 2.4$ and $\sim 2.5$, respectively, in the case of the structure, characteristic size has been probed by SAXS or by pore-size distribution by nitrogen adsorption.

The fine structure of the "secondary particle" developed in the obtaining of aerogels could be described as a surfacemass fractal with the correlated surface and mass fractal dimensions decreasing from $\sim 2.4$ to $\sim 2.0$ and $\sim 2.7$ to $\sim 2.5$, respectively, as the aerogel bulk density increases from $0.25\left(r_{w}=66\right)$ up to $0.91 \mathrm{~g} / \mathrm{cm}^{3}\left(r_{w}=6\right)$.

\section{ACKNOWLEDGMENTS}

Research was partially supported by LNLS-National Synchrotron Light Laboratory (Brazil) and FAPESP (Brazil). 
${ }^{1}$ C. J. Brinker and G. W. Scherer, Sol-Gel Science: The Physics and Chemistry of Sol-Gel Processing (Academic, San Diego, 1990).

${ }^{2}$ D. R. Vollet, D. A. Donatti, and A. Ibañez Ruiz, J. Non-Cryst. Solids 288, 81 (2001).

${ }^{3}$ D. R. Vollet, D. A. Donatti, and A. Ibañez Ruiz, Phys. Rev. B 69, 064202 (2004).

${ }^{4}$ S. J. Gregg and K. S. W. Sing, Adsorption, Surface Area and Porosity (Academic, New York, 1967).

${ }^{5}$ D. W. Schaefer and K. D. Keefer, Phys. Rev. Lett. 53, 1383 (1984).

${ }^{6}$ R. Vacher, T. Woignier, J. Pelous, and E. Courtens, Phys. Rev. B
37, 6500 (1988).

${ }^{7}$ G. Porod, in Small Angle X-ray Scattering, edited by O. Glatter and O. Kratky (Academic, London, 1982).

${ }^{8}$ D. Rojanski, D. Huppert, H. D. Bale, Xie Dacai, P. Schmidt, D. Farin, A. Seri-Levy, and D. Avnir, Phys. Rev. Lett. 56, 2505 (1986).

${ }^{9}$ G. W. Scherer, D. M. Smith, and D. Stein, J. Non-Cryst. Solids 186, 309 (1995).

${ }^{10}$ P. Pfeifer, F. Ehrburger-Dolle, T. P. Rieker, M. T. González, W. P. Hoffman, M. Molina-Sabio, F. Rodríguez-Reinoso, P. W. Schmidt, and D. J. Voss, Phys. Rev. Lett. 88, 115502 (2002).

${ }^{11}$ H. D. Bale and P. W. Schmidt, Phys. Rev. Lett. 53, 596 (1984). 